\title{
Serial learning and transfer in rats: Effects of changes in stimulus-stimulus associations, pattern structure, and serial position information
}

\author{
STEVEN J. HAGGBLOOM \\ Arkansas State University, State University, Arkansas
}

\begin{abstract}
Four groups of rats ( $n=6$ per group) were trained in a runway on a serial learning task. Groups were treated identically in Phase 1, receiving two daily presentations of a five-element series consisting of decreasing numbers of .045-g food pellets over successive runs, for example, 14-7-31-0. All groups learned to anticipate, and run slowly to, the terminal 0-pellet element, behavior that has been attributed to learning of a less-than rule, stimulus-stimulus (S-S) associations, and knowledge of the serial position of items. In Phase 2, subjects were transferred to one of four test series: 20-14-7-3-0, 20-7-3-14-0, 20-14-7-3-1-0, or 20-7-14-3-1-0. Anticipation was disrupted on the first two series, which maintained the integrity of serial position information and in the first case the less-than rule, but eliminated the terminal portion of the associative chain. Anticipation was unimpaired by transfer to either of the last two series. These series maintained the integrity of the terminal 3-1-0 portion of the associative chain but presented altered information about serial position, and in the last case also altered the less-than rule. The results, which supported the memory-discrimination model of rat serial learning, are discussed with reference to related transfer experiments in human serial learning.
\end{abstract}

Investigations of serial anticipation learning in rats have employed patterns or lists consisting of different numbers of .045-g food pellets as elements. Under some conditions rats learn to anticipate one or more elements of a series, anticipation usually being indexed by slower running to small food quantities, especially 0 food pellets or nonreinforcement, than to larger quantities. For example, on the series 14-7-3-1-0, employed by Hulse and Dorsky (1977), rats learned to run much slower on Run 5, in anticipation of the terminal 0-pellet element, than on earlier runs in the series.

Three different models have been proposed to account for serial anticipation behavior in rats. According to the memory-discrimination model (e.g., Capaldi \& Molina, 1979; Capaldi, Nawrocki, \& Verry, 1982), each pattern element comes to be signaled by the rat's memory of one or more prior elements in the series; that is, rats learn a series of stimulus-stimulus (S-S) associations, perhaps including remote associations (Capaldi, 1985). In this view, learning to anticipate, say, the terminal element of a series depends on the discriminability of the element(s) signaling 0 pellets from other elements, especially from those signaling larger food quantities. Experiments have demonstrated that element discriminability is a factor regulating serial anticipation learning by rats (e.g., Capaldi \& Molina, 1979; Capaldi et al., 1982; Haggbloom \& Brooks, 1985).

These experiments were supported by a Faculty Research Grant to the author from Arkansas State University. Requests for reprints should be addressed to the author, Department of Psychology, Arkansas State University, P. O. Box 2127, State University, AR 72467-2127.
The rule-learning model (e.g., Hulse, 1978) proposes that, at least under some conditions, rats induce a rule governing the structure of a serial pattern, and anticipation of one element is regulated by application of the rule to some representation of the preceding element(s). According to this view, ease of serial learning is related to pattern complexity, because the more complex the pattern, the more difficult it will be to discover the rule. Experiments have demonstrated that complex patterns are sometimes learned more slowly than simple patterns (e.g., Hulse \& Dorsky, 1977).

Recently, Roitblat, Pologe, and Scopatz (1983) suggested that whatever else rats learn in a serial anticipation task, that is, rules or S-S associations, they also come to represent the elements as items in serial position. In other words, they have knowledge about elements' serial position in the list. Roitblat et al. (1983, Experiment 4) trained rats on the 14-7-3-1-0 series and then presented them with test patterns on which the 0-pellet element was substituted for the elements in positions 1 to 4 over a series of test trials, that is, 0-7-3-1-0, 14-0-3-1-0, 14-7-0-10 , and 14-7-3-0-0. Substitution of 0 pellets for reinforcement in any position provided an unfamiliar cue signaling the next run, but nevertheless did not disrupt performance. According to Roitblat et al., the representation of items in serial position does not depend on the integrity of S-S associations or the integrity of the rule governing the pattern structure. Thus, once the pattern is learned, and the elements are represented as items in serial position, that is, as a list, anticipation behavior is not adversely affected by missing elements. Knowledge about an item's 
serial position provides sufficient information for correct anticipation behavior.

The research strategy employed here was similar to that used by Roitblat et al. (1983). After training on the 14-7-3-1-0 series, subjects were transferred to one of four test series. Group names indicate whether subjects could employ S-S associations (S-S), the less-than rule (R), knowledge of items' serial position $(\mathrm{P})$, or some combination of these strategies when presented with the transfer patterns. Thus, Group P/R was tested on the pattern 20-14-7-3-0. Removal of the 1-pellet element from the pattern alters what could be a critical S-S association, but the pattern leaves intact serial position information $(P)$ (i.e., the 0-pellet element remains in the fifth position), and the less-than rule ( $R$ ) remains in effect, because each element is still smaller than the one it follows. A disruption in performance here during transfer would indicate the importance of the 1-pellet element, either alone or as an integral part of a series of S-S associations, as a signal of 0 pellets. On the other hand, anticipation behavior regulated by knowledge of serial position or application of a less-than rule should not be affected by this pattern alteration. A second group, Group P, was employed to assess whether anticipation behavior could be maintained by knowledge of serial position alone. Group $P$ experienced a shift to the pattern 20-7-3-14-0. This pattern alters both S-S associations and the less-than rule, but maintains the integrity of the location of the 0-pellet element in the list.

Two additional groups experienced patterns that altered the location of the 0 -pellet element in the list, moving it to the sixth position, but maintained the integrity of critical S-S associations at the end of the chain, that is, 3-1-0. In the case of Group S-S/R, given the pattern 20-14-7-3$1-0$, the integrity of the less-than rule was also maintained. In the case of Group S-S, given the pattern 20-7-14-3-10 , the less-than rule was altered. Behavior in Groups S-S/R and S-S should be disrupted to the extent that anticipation is regulated more by knowledge of serial position and/or the less-than rule than by S-S associations.

\section{METHOD}

\section{Subjects}

The subjects were 24 male rats, approximately 90 days old at the beginning of the experiment, bred in the laboratory from Holtzman stock.

\section{Apparatus}

Rats were tested in a straight enclosed runway modeled after that used by Hulse and Dorsky (1977). The runway was $92 \mathrm{~cm}$ long, with a $30-\mathrm{cm}$ start box and a $30-\mathrm{cm}$ goal area separated from the rest of the runway by manually operated guillotine doors. The goal area contained an $11-\mathrm{cm}$ niche at a right angle to the runway and an unpainted goal cup positioned against the end wall of the goal area. The goal cup was constructed by drilling a $6-\mathrm{cm}$ diameter hole in a small block of wood. The inside width of the runway was $10 \mathrm{~cm}$ and the inside height was $11 \mathrm{~cm}$. The runway was painted flat black throughout. The start and goal areas were covered with hinged Plexiglas, and the runway proper was covered with hardware cloth. Raising the guillotine door between the start box and the runway triggered a .01-sec timer, which was stopped when the rat entered the goal box and interrupted a photobeam $20 \mathrm{~cm}$ into the goal area. Food reinforcement consisted of an appropriate number of $.045-\mathrm{g}$ Noyes pellets.

\section{Procedure}

The experiment was conducted in two identical replications. The procedures described below are for a single replication. Fourteen days prior to the beginning of training, the rats were placed on a food deprivation schedule, consisting of $12 \mathrm{~g}$ of food per day, which was maintained throughout the experiment. The amount of food received in the goal box was subtracted from the daily ration. Water was always available. On Days 12-14 of deprivation, the rats were tamed by handling them in squads of four for $2 \mathrm{~min}$ per squad. After being handled, the rats were returned to their home cages and fed $10.045-\mathrm{g}$ food pellets from a small dish placed in the cage.

On Day 15 of deprivation, 3 rats $(6$, combining the two replications) were randomly assigned to each of four groups and Phase 1 training began. On each day of training, each rat received two trials, each trial consisting of five runs separated by a 10-15 sec interrun interval, on the series 14-7-3-1-0, in which the numbers represent the quantity of $.045-\mathrm{g}$ food pellets.

There were 13 days of training in Phase 1 , followed immediately by 2 days of testing with two pattern presentations, or test trials, on each test day. Groups $P / R$ and $P$ were shifted to the five-element test series 20-14-7-3-0 and 20-7-3-14-0, respectively. Groups S-S/R and $S-S$ were shifted to the six-element test series $20-14-7-3-1-0$ and 20-7-14-3-1-0, respectively.

Throughout training and testing, subjects were taken to the experimental room in squads of 4 , containing 1 rat from each group. Each subject within the squad was administered Trial 1, with the order of running subjects in a squad determined randomly each day. After each subject in a squad had received Trial 1, Trial 2 was administered according to the same order. The interval between successive trials, or pattern presentations, was about 10-15 min. Rats were removed from the goal box immediately after consuming the reinforcement, or after $10 \mathrm{sec}$ in the case of nonreinforcement. If a rat failed to complete a run within $60 \mathrm{sec}$, it was placed in the goal box and $60 \mathrm{sec}$ was recorded as the time for that run.

\section{RESULTS}

Running times to each pattern element on the last day of Phase 1 are presented in the left panel of Figure 1 for Groups P/R and P and in the left panel of Figure 2 for

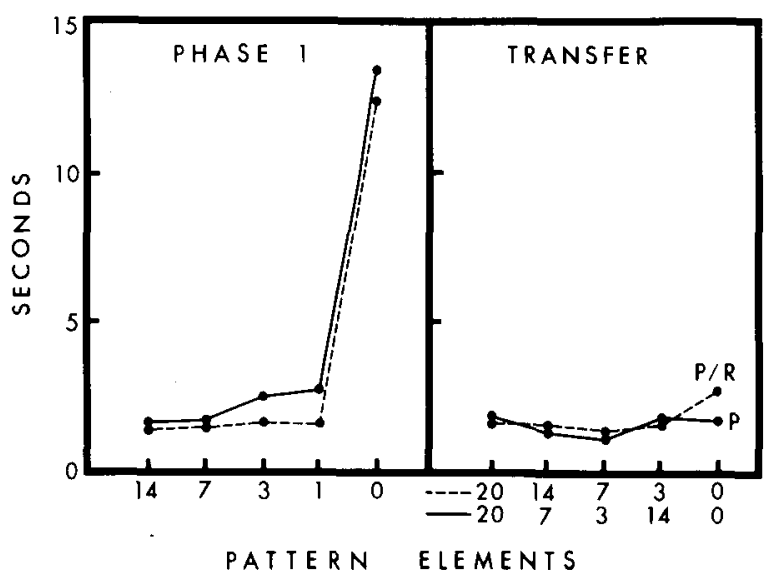

Figure 1. Running times for Groups $P / R$ and $P$ to each pattern element on the last day of Phase 1 (left panel) and on both test days (right panel). 


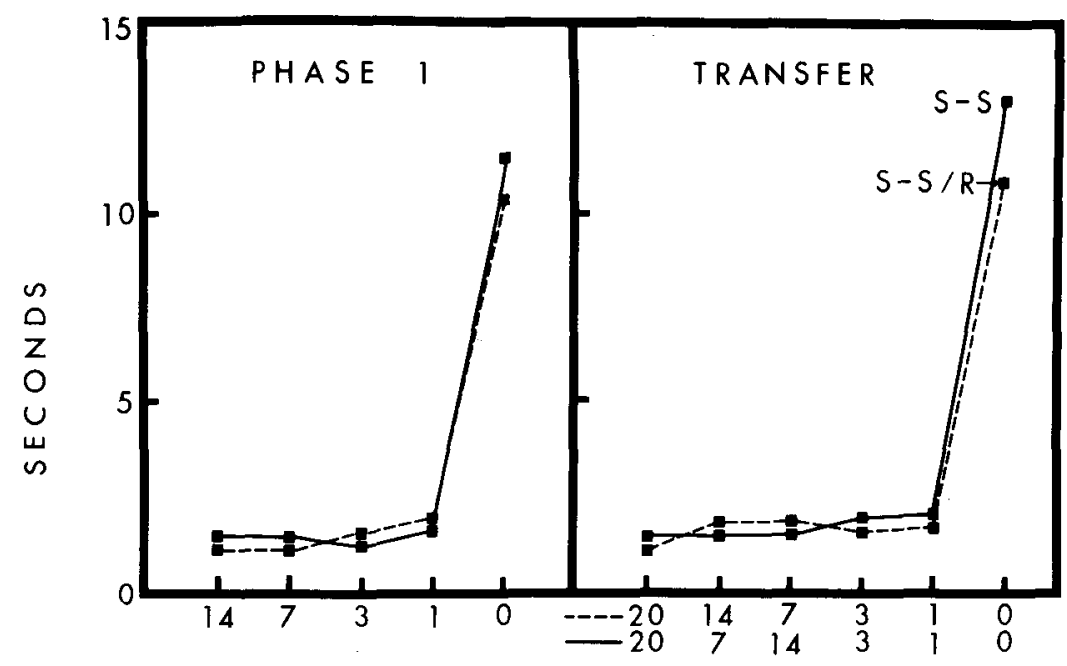

PATTERN ELEMENTS

Figure 2. Running times for Groups S-S and S-S/R to each pattern element on the last day of Phase 1 (left panel) and on both test days (right panel).

Groups S-S/R and S-S. All four groups were treated identically in Phase 1 , and as a comparison of the two figures shows, their performance was essentially the same. Each group learned to anticipate, and run slower to, the terminal 0-pellet element than to earlier elements of the series. An analysis of variance (ANOVA) with groups and replications as between-subjects variables and runs and trials as within-subject variables yielded a highly reliable main effect of runs $[F(4,64)=19.61, p<.001]$, but no groups $X$ runs interaction $[F(12,64)=1.30]$. All groups ran slower on Run 5 than on Run 1 [smallest $F(1,64)=27.68$ in Group S-S/R].

The right panel of Figure 1 shows the performance, averaged over both test days, of Groups $P / R$ and $P$ on each of the five elements of their respective transfer series. As can be seen, anticipation of the 0-pellet element was eliminated in Group $P$, which was given a transfer series in which S-S associations and the less-than rule were both altered and only serial position information was left intact. Anticipation behavior was similarly eliminated in Group P/R, for which the transfer series left both serial position information and the less-than rule intact, but altered S-S associations. An ANOVA applied to the data shown in the right panel of Figure 1 showed no differences due to groups $(F<1)$, runs $[F(4,32)=1.02]$, or groups $\times$ runs $[F(4,32)=1.37]$. Anticipation of the 0 pellet element showed no sign of improvement over the four transfer trials in either group. Mean group running times to the 0 -pellet element over the four test trials were $2.56,2.25,1.26$, and $1.33 \mathrm{sec}$ in Group $P$ and $2.75,1.37$, 5.26 , and $1.69 \mathrm{sec}$ in Group P/R. Neither the runs $\times$ trials nor the groups $x$ runs $\times$ trials interaction was significant $(\mathrm{Fs}<1)$.

The right panel of Figure 2 shows the performance, averaged over both test days, of Groups S-S/R and S-S on each of the six elements of their respective transfer series. As can be seen, Group S-S/R, for which serial position information was altered but the terminal part of the $S-S$ associative chain and the less-than rule remained intact, anticipated the terminal 0-pellet element about as well as at the end of Phase 1. Moreover, Group S-S, for which only the 3-1-0 portion of the associative chain remained intact, the less-than rule and serial position information being altered, also anticipated the 0 -pellet element as well as at the end of Phase 1 and as well as Group S-S/R. An ANOVA applied to the data shown in the right panel of Figure 2 yielded a highly reliable main effect of runs $[F(5,40)=9.25, p<.001]$, but no groups $\times$ runs interaction $[F(5,40)=1.13]$. Both groups ran substantially slower to the 0-pellet element than to the initial 20-pellet element [smallest $F(1,40)=17.62$ for Group S-S/R].

\section{DISCUSSION}

In the present experiment, four groups of rats received identical training in Phase 1 , receiving two daily trials of five runs each on the descending series of 14-7-3-1-0 food pellets. Each group learned to anticipate the terminal 0pellet element and ran markedly slower to it than to other elements of the series. This behavior has been attributed to rats' knowledge of a less-than rule (Hulse, 1978; Hulse \& Dorsky, 1977), to S-S associations established between elements, perhaps including remote associations (Capaldi, 1985; Capaldi \& Molina, 1979), and to knowledge about the serial position of the 0-pellet element in the list (Roitblat et al., 1983).

Following Phase 1, each group was shifted to a transfer series designed to interfere with the rats' use of S-S associations, rules, serial position information, or some combination of these strategies. Groups S-S and S-S/R 
experienced transfer series that maintained the integrity of the terminal 3-1-0 portion of the S-S associative chain. In Group S-S/R, the integrity of the less-than rule was also maintained. Anticipation of the 0-pellet element in the transfer series was equally good in both groups and was essentially the same as at the end of Phase 1 , a finding that suggests that neither knowledge of the serial position of the 0-pellet element nor the less-than rule was essential to good performance. It is conceivable that the 14-3-1-0 portion of the transfer series experienced by Group S-S was sufficient to provoke application of the less-than rule, and so this series may fail to distinguish between the rule-learning and S-S association models. On this basis, however, one would also have to predict substantial positive transfer in Group P/R, which, as noted below, did not occur.

Groups $\mathrm{P}$ and $\mathrm{P} / \mathrm{R}$ experienced transfer series that altered the terminal 1-0 link in the associative chain, but maintained the integrity of serial position information. The integrity of the less-than rule was also maintained in Group P/R. In both cases, anticipation of the 0 -pellet element was eliminated, indicating that the presence of the 1-pellet element as the terminal link in the associative chain is essential to anticipation behavior, but that neither the less-than rule nor knowledge of serial position provides sufficient information for correct responding.

The present finding that removal of the 1-pellet element from the end of the series disrupted anticipation behavior would appear to be inconsistent with the finding, reported by Roitblat et al. (1983, Experiment 4), that substitution of the 0-pellet element for other elements in the series did not disrupt anticipation behavior. However, Roitblat et al.'s subjects had never previously been required to respond immediately after receiving the 0 -pellet element, and there is no clear basis for predicting any particular behavior change to be produced by that substitution. In contrast, the subjects in the present study evidently learned to run slowly following presentation of the 1-pellet element, and so its removal from the series resulted in fast running to the 0-pellet element. It remains unclear why the substitution of 0 pellets for other elements in the series had no behavior effect in Roitblat et al.'s experiment, but the finding of no difference hardly recommends the view that the elements were represented as items in serial position. Moreover, even if the series is represented as a list, such a representation clearly does not make performance "impervious to any distraction from missing elements" (Roitblat et al., 1983, p. 495).

The finding here that removal of the 1-pellet element disrupted anticipation of 0 pellets even when the integrity of the less-than rule was maintained (Group P/R) suggests that correct application of the rule to predict 0 pellets requires some knowledge over and above knowledge of the rule. Since the integrity of serial position information was also maintained in Group $P / R$, the extra knowledge required must not be about the serial position of the 0-pellet element in the list. If the extra knowledge were about the relationship between the 1 - and 0-pellet elements, however, that knowledge alone might generate anticipation of 0 pellets, and knowledge of the rule would be quite superfluous.

The transfer data reported here are in agreement with recent data, reported by Capaldi and co-workers (see Capaldi, 1985), indicating that rats form S-S associations and supporting a chaining hypothesis of serial learning. The chaining hypothesis predicts that a transfer task on which correct responding can be completely guided by S-S associations acquired during original learning will yield massive, perhaps $100 \%$, positive transfer, like that obtained in Groups S-S and S-S/R in the present study.

The transfer task employed here is reminiscent of the transfer methodology developed by Young and associates (e.g., Hakes, James, \& Young, 1964; Hakes \& Young, 1966; Young, Hakes, \& Hicks, 1965) in extensive tests of the chaining hypothesis in human serial learning. Young trained subjects on a serial list of the form A-B-C-D-E and then tested them on a derived paired-associates list, that is, B-C, A-B, D-E, C-D. Because all the associations needed to perform the paired-associates task were presumably formed during the initial stage of serial learning, the chaining hypothesis predicts massive positive transfer. Generally speaking, these experiments yielded results ranging from negative transfer to little or no transfer.

Young's data have been cited in some recent reports on animal serial learning as evidence against S-S associations (e.g., Roitblat et al., 1983; Straub \& Terrace, 1981). It now appears, however, that under typical experimental procedures, the derived paired-associates task not only will not yield massive positive transfer, but should not yield massive transfer, because a major component of the functional stimulus regulating anticipation in the serial task is missing in the paired-associates task.

Slamecka (1977) identified the missing link in the associative chain as a stimulus produced by the subject's correct anticipatory response. Following a correct response in the serial task, for example, when the subject correctly says " $B$," the stimulus that signals item $C$ is a compound stimulus consisting of the B that appears in the window of the memory drum and a stimulus produced by the subject's response "B." During training on the transfer task, the response-produced cue that formerly was part of the stimulus complex that signaled the correct response is absent, and, in fact, is replaced by a response-produced cue that signals a different response. For example, on the derived list B-C, A-B, D-E, $C-D$, a subject who responds " $C$ "' to the stimulus $B$ would then have the response-produced cue " $C$ "' that signals $D$ and the stimulus $\mathrm{A}$ on the memory drum that signals $\mathbf{B}$.

Slamecka (1977) found that a derived list that did not place response-produced cues in conflict with the cue provided by the memory drum, that is, A-B, B-C, C-D, $D-E$, yielded massive positive transfer, $94 \%$ of the maximum possible transfer on one measure. Thus, in fact, human serial learning data are not inconsistent with the chaining hypothesis or the notion of S-S associations upon 
which that hypothesis rests, when, as Slamecka (1977) has shown, there is "genuine associative compatibility between the tasks" (p. 227).

Young (1962) used a transfer design even more like the one used here, in which subjects were trained on a serial list and tested on a derived serial list with some items in the same serial position as in the original list, and others randomly rearranged. The major finding was that items in unchanged serial positions were easier to learn than items in changed serial positions. The results were taken as support for a position-cue hypothesis of serial learning. Young's (1962) results, however, would also be predicted by a version of the chaining hypothesis, called the bundle hypothesis (Capaldi, 1985; Slamecka, 1985), that postulates S-S associations between remote items as well as between adjacent items. Remote associations would be stronger, on average, between items in a constant serial position than between randomly rearranged items. Groups $S-S$ and $S-S / R$ in the present experiment experienced transfer series in which the position of items was changed but the integrity of S-S associations, both adjacent and remote, was maintained. Positive transfer in these groups was massive. A similar transfer test could be used to determine the relative control exercised by position cues and remote S-S associations in Young's (1962) derived serial list situation. In addition to Young's groups, the test could employ, for example, a group transferred from the serial list A-B-C-D-E-F-G-H to a derived serial list of the form D-A-F-C-H-E-B-G, in which items A, $\mathrm{C}, \mathrm{E}$, and $\mathrm{G}$ occupy changed serial positions but the remote associations A-C, C-E, and E-G remain intact.

As the foregoing discussion illustrates, Young (1962) tested an impoverished version of the chaining hypothesis, one that did not acknowledge the potential significance of remote associations. Historically, arguments against the chaining hypothesis have ignored remote associations, and it is just such an impoverished chaining hypothesis that was deemed inadequate by Hulse and Dorsky (1977), Roitblat et al. (1983), and Straub and Terrace (1982). On the other hand, Capaldi (1985) presented considerable evidence for remote associations in animal serial learning, and Slamecka (1985) reviewed evidence for remote associations in human serial learning. An impoverished chaining hypothesis may indeed be inadequate to account for human and animal serial learning data, but, as
Slamecka (1985) pointed out, "what has been completely overlooked is the vital presence of remote associations" (p. 420).

\section{REFERENCES}

CAPAldi, E. J. (1985). Anticipation and remote associations: A configural approach. Journal of Experimental Psychology: Learning, Memory, \& Cognition, 11, 444-449.

CaPaldi, E. J., \& Molina, P. (1979). Element discriminability as a determinant of serial pattern learning. Animal Learning \& Behavior, 7, 318-322.

Capaldi, E. J., Nawrocki, T. M., \& Verry, D. R. (1982). Difficult serial anticipation pattern learning in rats: Rule-encoding vs. memory. Animal Learning \& Behavior, 10, 167-170.

HagGbloom, S. J., \& BRooKs, D. M. (1985). Serial anticipation and pattern extrapolation in rats as a function of element discriminability. Animal Learning \& Behavior, 13, 303-308.

Hakes, D. T., James, C. T., \& Young, R. K. (1964). A re-examination of the Ebbinghaus derived-list paradigm. Joumal of Experimental Psychology, 68, 508-514.

HAKes, D. T., Young, R. K. (1966). On remote associations and the interpretation of derived-list experiments. Psychological Review, 73, 248-251.

Hulse, S. H. (1978). Cognitive structure and serial pattern learning by animals. In S. H. Hulse, H. Fowler, \& W. K. Honig (Eds.), Cognitive processes in animal behavior (pp. 311-340). Hillsdale, NJ: Erlbaum.

Hulse, S. H., \& DorsKy, N. P. (1977). Structural complexity as a determinant of serial pattern learning. Learning \& Motivation, 8, 488-506.

Roitblat, H. L., Pologe, B., \& Scopatz, R. A. (1983). The representation of items in serial position. Animal Learning \& Behavior, 11, 489-498.

Slamecka, N. J. (1977). A case for response-produced cues in serial learning. Journal of Experimental Psychology: Human Learning \& Memory, 3, 222-232.

Slamecka, N. J. (1985). Ebbinghaus: Some associations. Journal of Experimental Psychology: Learning, Memory, \& Cognition, 11, 414-435.

Straub, R. O., \& Terrace, H. S. (1981). Generalization of serial learning in the pigeon. Animal Learning \& Behavior, 9, 454-468.

YounG, R. K. (1962). Tests of three hypotheses about the effective stimulus in serial learning. Journal of Experimental Psychology, 63, 307-313

Young, R. K., Hakes, D. T., \& Hicks, R. Y. (1965). Effects of list length in the Ebbinghaus derived-list paradigm. Joumal of Experimental Psychology, 70, 338-341.

(Manuscript received April 16, 1985; revision accepted for publication September 23, 1985.) 